Ivan Jovanović*

Faculté de Philosophie, Université de Niš
UDK : 811.133.1'37:811.163.41

DOI: $10.19090 /$ gff.2021.3.105-117

originalni naučni rad

\title{
LES NOMS ALTERNATIFS DE L'ORGANE SEXUEL FÉMININ EN FRANÇAIS ET EN SERBE : ASPECT LEXICO-SÉMANTIQUE**
}

Dans le présent article nous procédons à l'analyse des noms alternatifs de l'organe sexuel féminin en français et en serbe afin de montrer toutes les similitudes et les différences qui se produisent au niveau lexico-sémantique. En partant des postulats de l'analyse sémique et conceptuelle (Ullmann, 1951 ; Touratier, 2010 ; Lakoff et Johnson, 1980) ainsi que de la classification de Guiraud (1995) des unités lexicales obscènes, notre corpus est répertorié selon les formes de polysémie induisant la signification des noms alternatifs (relations métaphoriques basées sur la forme, la fonction, la couleur et les sèmes de l'expression collective) et selon les types de métaphore conceptuelle à partir desquels l'organe sexuel féminin est conceptualisé comme un bâtiment, un lieu, un animal, une plante ou un espace géographique. Le corpus de notre recherche se compose des dictionnaires obscènes français et serbe de Pierre Guiraud (1995), Alfred Delvau (1997), Robert Édouard (2004), Danko Šipka (2011), Petrit Imami (2007), ainsi que des dictionnaires d'argot d'Abdelkarim Tengour (2013), Dragoslav Andrić (1976) et Borivoje Gerzić (2010).

Mots-clés : noms alternatifs, sexe féminin, aspect lexico-sémantique, langue française, langue serbe.

\section{INTRODUCTION}

Le corps humain représente le siège unique de toutes nos sensations. Toutes nos fonctions psychiques, en particulier, sont créées et dénommées d'après des images corporelles. Les trois grandes fonctions psychiques, l'intelligence, l'affectivité et la volonté, sont respectivement liées à la tête, au ventre et au sexe, organes dont les fonctions symbolisent notre activité

\footnotetext{
*ivan.jovanovic@filfak.ni.ac.rs

** Cet article est rédigé dans le cadre du projet scientifique Les langues, les littératures et les cultures romanes et slaves en contact et en divergence ( $\mathrm{N}^{\circ}$ 81/1001-13-01) soutenu par la Faculté de philosophie de l'Université de Niš, l'AUF (Agence universitaire de la Francophonie) et l'Ambassade de France en Serbie.
} 
psychologique et notre relation au monde (Guiraud, 1975 : 8). La question du sexe et de la sexualité ${ }^{1}$ est étroitement liée à l'érotisme qui représente essentiellement un langage, un système de signes et une symbolique. Cependant, Guiraud sépare la sexualité de l'érotisme en soulignant que la sexualité renvoie à un aspect charnel tandis que l'érotisme touche plutôt à l'aspect spirituel. La sexualité a pour fin la reproduction de l'espèce alors que le but de l'érotisme est le plaisir qui n'est que le moyen et le mécanisme placé par la nature au service de la sexualité (Guiraud, 1978 : 107). L'étude des formes symboliques et métaphoriques de la sexualité est incomplète sans l'examen de son propre vocabulaire, des mots et des images au moyen desquels elle s'exprime dans le contexte érotique. Il n'est guère de verbe actif, et plus particulièrement de verbe transitif, ni de nom d'agent, d'instrument ou de patient, qui ne contienne une image sexuelle en puissance, prête à se concrétiser.

Quant au sexe de la femme qui fait l'objet de notre étude, la littérature érotique, non plus que le langage populaire, ne distingue la "vulve» et le «vagin», désignés par l'unique con. Certes, le contexte et l'étymologie permettent de distinguer parmi les innombrables synonymes la "vulve ${ }^{2}$ » (écartée), le «vagin ${ }^{3}$ » (pénétré), le "pubis» (poilu), le «clitoris» (protubérant), mais d'une façon générale cet organe si complexe est vu d'une façon très peu différenciée. Ceci tient aussi bien à des tabous qu'à des raisons anatomiques évidentes, mais il n'en reste pas moins que le langage montre que

${ }^{1}$ Le vocabulaire de la sexualité se caractérise par une fécondité verbale tout à fait exceptionnelle et qui lui confère une place unique dans le système de la langue; le moindre concept y est représenté par des centaines de synonymes. À titre d'exemples, le « coït » est désigné en français par 1300 synonymes et en serbe par 800, le « pénis » et « le vagin » par 600 en français et par 246 et 220 en serbe. Étant donné que les trois concepts sont intégrés dans un même système lexical : le forgeron frappe sur son enclume avec son marteau, le cavalier attaque la forteresse sabre au clair, il en résulte un ensemble de plus de 3000 mots et expressions pour désigner une même notion. Et cet ensemble est loin d'être limitatif. Cet inventaire pourrait être doublé ou triplé et c'est presque tout le vocabulaire qui, le contexte aidant, peut être attiré dans ce système (Guiraud, $1995: 13 ; 1978: 116$; Шипка, $2011: 158,160$ ).

2 La vulve provient du mot latin vulva et désigne " une enveloppe », formée par les lèvres (Guiraud, 1995 : 38).

${ }^{3}$ Guiraud souligne qu'étymologiquement, le vagin tire son origine du mot latin vagina et désigne « gaine » et « un fourreau » $(1995: 38)$. 
les hommes connaissent mal l'anatomie de leur compagne, ce que confirment les sexologues (Guiraud, 1995 : 38).

\section{CADRE THÉORIQUE, MÉTHODOLOGIQUE ET CORPUS DE LA RECHERCHE}

Dans le cadre de notre recherche, nous partons des postulats de la sémantique lexicale, plus précisément de la métaphore lexicale et de la sémantique cognitive dont la partie intégrante est la métaphore conceptuelle.

Dans la sémantique et la linguistique traditionnelles, la métaphore est généralement comprise comme l'un des mécanismes les plus courants de la polysémie, grâce auquel un grand nombre de nouvelles significations de mots sont induites dans le lexique d'une langue. Ainsi, selon de nombreux auteurs tels que Gortan-Premk (2004 : 86), Ullmann (1952 : 202), Touratier (2010 : 119), Поповић (2009 : 134), Tamba-Metz (2012: 41), la métaphore lexicale implique le transfert de nomination d'un terme (référent) à un autre, c'est-àdire d'un contenu sémantique à un autre, motivé par une connexion analogue de composants sémantiques de rang inférieur, avec remplacement obligatoire de l'archisème du contenu sémantique initial par l'archisème du contenu cible. Ainsi, la métaphore lexicale représente le transfert de noms d'un terme à un autre sur la base d'une certaine similitude entre eux. Autrement dit, chaque métaphore contient trois composants : l'idée à nommer, celle qui lui donne son nom et le trait de ressemblance qui contribue au rapprochement.

En plus de la métaphore lexicale, nous traitons également dans notre recherche sur la métaphore conceptuelle. En effet, les concepts qui règlent notre pensée structurent ce que nous percevons, la façon dont nous nous comportons dans le monde et dont nous entrons en rapport avec les autres. Notre système conceptuel joue ainsi un rôle central dans la définition de notre réalité quotidienne. Lakoff et Johnson (1985 : 14) émettent l'hypothèse que la plus grande partie de notre système conceptuel ordinaire est de nature métaphorique et ils ont trouvé un moyen de commencer à déterminer dans les détails quelles sont les métaphores qui structurent notre manière de percevoir, de penser et de faire. Dans notre article, nous considérerons les métaphores lexicale et conceptuelle comme des réalisations différentes d'un même processus, qui sont en principe égales et ne se manifestent qu'à des niveaux différents.

En ce qui concerne le cadre méthodologique de notre travail, nous avons appliqué la méthodologie relative à l'analyse contrastive. Selon Selena 
Stanković (2012 : 382), l'analyse contrastive est le nom d'une méthode linguistique issue de la linguistique structurale, d'une part, et de la linguistique anthropologique et comportementale, d'autre part, qui révèle des similitudes et des différences explicites dans la description comparative systématique de deux ou plusieurs langues.

Le corpus de notre recherche est constitué des dictionnaires obscènes français et serbe de Pierre Guiraud (1995), Alfred Delvau (1997), Robert Édouard (2004), Danko Šipka (2011), Petrit Imami (2007), ainsi que des dictionnaires d'argot d'Abdelkarim Tengour (2013), Dragoslav Andrić (1976) et Borivoje Gerzić (2010). Dans notre recherche, le corpus est répertorié selon les analogies qui s'effectuent entre les unités lexicales. Dans la plupart des cas c'est sur les niveaux de forme, de fonction, de couleur et des sèmes de l'expression collective. À ce propos, notre objectif est de fournir et d'analyser l'inventaire des noms alternatifs de l'organe sexuel féminin, 108 en français et 33 en serbe, à partir des analogies métaphoriqes et concepts donnés, afin de dégager toutes les similitudes et les différences qui apparaissent au niveau lexico-sémantique. Il est à noter que nous avons pris en considération uniquement les unités lexicales qui sont en usage fréquent tandis que celles, étant vieillies ou tombées en désuétude, ne font pas l'objet de notre étude.

\section{ANALYSE DU CORPUS}

Analogie de forme

L'analogie de forme est la plus répandue dans notre corpus étant donné que le sexe de la femme ressemble à de nombreux objets ronds et ovales et il est perçu comme : a) un vaisseau, un ustensile creux : le navire, la nacelle, le vase, le bassin, le baquet, le seau, la marmite, le pot, le chaudron, l'écuelle, le bénitier, la bouteille, le cornet, le creuset, le sac, le bissac, la bourse, la blouse, le panier, la boîte d'amourette, la gouttière, le puits, la cheminée, le moule, le bonnet, le chapeau, капа, шубара, чизма, буре, шоља, мешалица; b) un creux naturel : la brèche, la fente, le joint, le perc, la raie, la plaie, le fossé, la fosse, le vallon, la vallée, la grotte, le terrier, la tanière, la garenne, le clapier, јамa, пукотина, рака, рана, рупа, рупица, рупингер, септичка јама, шоља, тунел ; c) une ouverture : l'entrée, le passage, la porte, la porte de devant, le portail, le huis, la grille, la serrure.

Le navire et la nacelle désignent différents types de bateau (Rey, 2006 : 2335 ; 2353) qui figurément ressemblent au vagin d'après leur aspect ovale, 
allongé et creux de surface. De telles métaphores proviennent du domaine maritime. Les lexèmes : le vase, le bassin, le baquet, le seau, la marmite, le pot, le chaudron, l'écuelle, le bénitier, la bouteille sont des objets, de différentes tailles, avec une entrée par laquelle l'on introduit un liquide. Métaphoriquement, ces objets s'identifient au sexe de la femme étant considéré également comme l'organe rempli de fluide. Dans le cadre des mots cités, la base de la métaphore sont les sujets culinaires (la marmite ${ }^{4}$, le pot, le chaudron, l'écuelle ${ }^{5}$, le puits ${ }^{6}$ ), hydrographe (le vase, le bassin, le baquet, le seau, la bouteille, gouttière ${ }^{7}$ ), religieux (le bénitier). À la différence de ces objets contenant un liquide, les objets suivants, référant métaphoriquement au vagin, se remplissent d'une matière solide, ce qui peut impliquer l'acte sexuel : le cornet ${ }^{8}$, le creuset, le sac $^{9}$,

${ }^{4} \mathrm{Il}$ est à souligner que les sèmes des sémèmes marmite, pot et chaudron se combinent. Ainsi le vagin qui est une fente suggère l'image de l'un de ses objets cités ci-dessus qui peuvent être fendu pour constater que ceci n'enlève rien à son utilité. Ce que traduit le jeu de mot: a) Bien que sa marmite soit fendue, Tabarin sait bien qu'elle ne cassera jamais ; b) Les femmes sont craintives parce que leur pot étant déjà fendu, au moindre bruit elles craignent qu'on ne le leur vienne casser (Guiraud, 1978 : 117).

${ }^{5}$ L'image de l'écuelle qui désigne ordinairement la sébille du " gueux » ou mendiant professionnel, combine le thème de l'écuelle " ustensile de cuisine", avec celui de la pasture (provende, avoine, picotin) due à la femme; et celui de l'objet sexuel dévalorisé et placé au plus bas échelon de l'échelle sociale, sous les espèces de la compagne du gueux : gueuse, coquine, truande (Guiraud, 1978 : 117).

${ }^{6} \mathrm{Au}$-delà de son sens primaire "puits d'eau vive ", ce terme connaît de nombreuses extensions sémantiques par lesquelles on trouve une métaphore sexuelle. A. Rey (2006 : 3007) note qu'un puits est un creux en cuisine au centre d'un mets et que l'expression puits d'amour désigne une espèce de pâtisserie et avait préparé ce sens.

${ }^{7} \mathrm{Ce}$ mot a désigné au départ la partie inférieure d'un toit, d'où l'eau tombe goute à goute (Rey, 2006: 1618). Par extension, gouttière se dit d'une fissure par où l'eau pénètre goutte à goutte à l'intérieur d'une maison (2006: 1619). Dans ce cas, nous avons une double analogie : gouttière s'approche à la fois au vagin de par sa forme (fissure) et à l'acte sexuel de par sa fonction puisque le fait de faire pénétrer de l'eau dans la gouttière fait allusion au coït.

${ }^{8} \mathrm{P}$. Guiraud précise qu'au niveau de l'image contextuelle, le cornet étant un objet creux dans lequel on "trempe la plume», ce qui a le trait à l'acte sexuel (Guiraud, 1995 : 249). D'autre part, A. S. Lagail (1903 : 150) note que le cornet de glace est cunnilinctus dans lequel la vulve est serrée entre les pouces et les index écartés pour faire remonter la partie charnue dans laquelle le clitoris est alors enfui.

${ }^{9}$ D'après la locution en avoir plein son sac "plus qu'on ne peut en supporter ", avoir le sac plein se dit aussi d'une femme enceinte, ce qui aussi confirme d'idée de l'acte sexuel (Guiraud, 1995 : 560). 
le bissac, la bourse ${ }^{10}$, la blouse ${ }^{11}$, le panier ${ }^{12}$, la boîte d'amourette ${ }^{13}$, le moule le $^{14}$. Par exemple, dans un cornet on introduit une boule, ce qui fait allusion à la partie supérieure du sexe masculin. Somme toute, il s'agit d'une idée, d'une image de mettre quelque chose dedans. Les noms tels que le bonnet, le chapeau, la coiffe, капа, шубара sont issus des métaphores vestimentaires et représentent les éléments enfoncés sur la tête du pénis (Guiraud, 1995 : 216 ; Шипка, 2011 : 160). Le substantif serbe чизма renvoie figurément au sexe de la femme par son entrée, c'est-à-dire, surface creuse (Шипка, 1999 : 161). Le terme буре touche aussi au vagin grâce à l'analogie de forme. En effet, dans les vers suivant tirés d'une chanson populaire serbe :

Чеп набио буре затворио (le bouchon, il a introduit, le tonneau, il a fermé) Подрумџија он је дуго био (Pendant longtemps le cellier il était) Напише се моје муштерије (Mes clients se sont saoulés) Само Рака може да набије (Seul Raka peut enfoncer)

I. Jovanović (2019: 161) explique que l'organe sexuel masculin est nommé par le lexème čep (bouchon) et le sexe féminin par le nom bure (tonneau). Les relations métaphoriques sont établies sur la base de similitudes de forme : l'organe sexuel masculin ressemble à l'objet mentionné, tandis que l'organe sexuel féminin est perçu comme un vaisseau avec une ouverture fermée par un certain objet. Au sens figuré, l'image ci-dessus de tirer et de pousser un bouchon dans un tonneau implique l'acte sexuel.

10 Guiraud fait remarquer qu'en fonction du contexte donné, ce mot peut signifier le pénis ou les testicules (1995: 186).

11 Dans ce cas, l'analogie de forme repose sur la blouse en tant que la poche du billard, tel qu'il est encore pratiqué dans les pays anglo-saxons. Le billard avec sa blouse, ses billes et son billard ou queue donne lieu à d'innombrables plaisanteries érotiques (Guiraud, 1995 : 175).

12 L'usage de ce mot est motivé par l'expression avoir son panier percé au sens de « perdre son pucelage » (Guiraud, $1995: 476)$.

13 Selon Larousse (2010), une boîte d'amourette est le jeu de société qui semble n'avoir été inventé que pour donner des gages. Guiraud mentionne le terme boîte à ouvrage, objet féminin par excellence, connoté par le sens érotique de besogne, ouvrage (Guiraud, 1995 : 177).

14 Il faut souligner que le moule peut toucher au pénis lorsqu'il est plein (moule à chapeau, à chaussure) et quand il est creux il renvoie au vagin (moule à gâteaux) (Guiraud, 1995 : 456). 
Nombreuses sont les unités lexicales dans les deux langues métaphorisant le vagin comme un creux naturel. Leur sème générique est /fissure/ induisant la métaphore fondamentale du système : la brèche, la fente, la raie, la plaie, le fossé, le vallon, la vallée, la grotte, le terrier, la tanière, la garenne, le clapier, јама, пукотина, рупингер, септичка јама, тунел. Il s'agit des éléments naturels se différenciant, comme d'ailleurs des vagins, par taille (les uns sont plus petits : la brèche, la fente, la raie, la plaie, le fossé, рака, рана, pyna, рупица, d'autres sont plus grands: la fosse, le vallon, la vallée, la grotte), par forme (forme régulière: la fente, la raie, la plaie, le fossé, тунел, руna, pуnица et irrégulière : grotte, fente), ou par objectif (certains sont occupés par des animaux: le terrier, la tanière, la garenne, le clapier). Ces derniers se rapportent à l'habitat du lapin (Guiraud, 1995) et peuvent figurément impliquer le coït. Le lapin est la métaphore du pénis et l'image de faire entrer ou sortir le lapin de « sa maison » fait allusion aux rapports sexuels. Ainsi, il est intéressant de souligner que le syntagme nominal serbe: септичка јама s'associe au sexe de la femme qui est mal entretenue et qui émet des odeurs désagréables.

Le vagin peut être également métaphorisé comme une ouverture, ce qui représente son sème générique et déclenche l'analogie de forme : l'entrée, le passage, la porte, la porte de devant, le portail, le huis, la grille ${ }^{15}$, la serrure ${ }^{16}$.

\section{Analogie de fonction}

Le sexe de la femme peut s'identifier aux différents objets par analogie de fonction. Il s'agit, en général, d'une pièce à l'intérieur de laquelle on pénètre et dont la nature varie selon le contexte et la nature de l'occupant: la chambre, le réduit, le cabinet, la cuisine, le four, le grenier, la grange, le fenil, l'étable, la basse-cour, l'atelier, la boutique, la forteresse, la citadelle, la casemate, le champ de bataille, le temple, le sanctuaire, la chapelle, Temple, l'autel, le labyrinthe, Банка, кућа, рерна.

15 D'après des locutions techniques de la langue du jeu de paume : marquer une chasse «indiquer le point où s'est arrêté une balle » et, métaphoriquement, " relever chez quelqu'un une faute dont on peut profiter », la grille étant « la fenêtre carrée placée au bout du toit, au jeu de paume » (Guiraud, 1995 : 377).

16 Guiraud note que l'emploi du mot est en général complémentaire de clé et que c'est l'image dont la psychanalyse a relevé le caractère érotique (Guiraud, 1995 : 573). 
Nous souhaitons particulièrement ponctuer deux choses : a) la plupart de ces termes sont motivés par une image militaire et par une idée de faire l'assaut et d'occuper une espace, une pièce. C'est aussi une idée de combattre où l'amour est perçu comme un combat, une bataille. Par exemple, pour pouvoir envahir une forteresse, il faut un artilleur, son canon et une brèche. Guiraud (1978 : 120) considère que la métaphore militaire est si cohérente, si pertinente que tous les modes, moyens et phases du combat et toutes les phrases qui les expriment contiennent, en puissance, une image sexuelle; b) un certain nombre de mots reposent sur la métaphore religieuse. C'est parce que dans ce contexte on peut extraire les relations du vicaire avec ses paroissiennes, des moines avec les nonnes et les novices représentant aussi un thème intarissable d'un folklore érotique où chapelle rime avec chandelle ${ }^{17}$ et dans lequel " l'enseignement religieux recoupe le thème du sanctuaire, du Temple où l'on vient sacrifier au culte du dieu d'amour » (Guiraud, 1978 : 123).

\section{Analogie de couleur}

Au-delà des relations analogiques de forme et de fonction, nous avons trouvé un exemple dans la langue serbe où la relation entre les deux concepts s'établie sur la base de la similitude de couleur. Il s'agit d'un manipulateur figurant sur certains instruments ou appareils. Dans notre cas, l'organe sexuel féminin, plus précisément son intérieur, est rouge et ressemble à un bouton qui émet une lumière rouge lorsqu'il est allumé: црвен тастер : « Масираћу теби сисе док ти ноге горе висе, набићу ти калабастер у тај твоји црвен тастер » (Јовановић, $2019: 162)$.

\section{SÈMES DE L’EXPRESSION COLLECTIVE}

Contrairement aux exemples donnés jusqu'à présent, dans lesquels la composante sémantique induisant la métaphore contient des informations sur l'une des propriétés externes du dénotat ou des informations sur notre compréhension du dentotat, nous trouvons des cas où le contenu sémantique est imprégné de sèmes reflétant le résultat d'une expression collective. Gortan-

17 Il s’agit du désignatif du pénis. 
Premk (2004 : 107) souligne qu'il existe deux types fondamentaux d'associations métaphoriques basées sur un sème expressif ; le premier est celui dans lequel le lexème de départ est un zoonyme ou un phytonyme tandis que le lexème cible est un humain et/ou ses parties du corps ; l'autre est celui dans lequel le lexème initial et le lexème cible nomment une personne : a) animaux : chat, chatte, minou, angora, coquille, coquille Saint-Jacques, crevasse, crevette, écrevisse, faucon, hérisson, huitre, lapin, petit lapin, pigeon, rat, souris, oie, oiseau sans plumes, риба, рибица, мачка, мачкица, чавка, кока; b) plantes : abricot, abricot de la jardinière, abricot fendu, feuille de sauge, fève, figue, nénuphar, oignon, osière, rose, fleur, црна љубичица, смоква, шљива, жбун ; c) personne : Мица, Цана, Мицојка $\rightarrow$ vagin.

Les zoonymes et les phytonymes en tant que les lexèmes de départ ont dans leur contenu sémantique des sèmes qui sont le résultat d'une expression collective, la compréhension collective que les animaux comme chat, chatte, minou, риба, рибица, мачка etc. et les phytonymes tels que abricot, fève, figue etc. possèdent certaines propriétés relatives à l'homme, que l'on attibue à l'homme ou à une de ses parties somatiques. Gortan Premk (2004 : 108) souligne que toutes les métaphores qui partent des lexèmes nommant un homme ou ses parties sont expressivement non marquées, dans notre cas Mica, Cana $\rightarrow$ vagin, et toutes les métaphores qui partent des choses, des plantes et des animaux et finissent par nommer un homme ou ses parties sont expressives : oie $\rightarrow$ vagin, čavka $\rightarrow$ vagin.

\section{MÉTAPHORE CONCEPTUELLE}

Contrairement à la sémantique et à la linguistique traditionnelles, la linguistique moderne, et en particulier celle basée sur une approche cognitiviste, place la métaphore dans le processus de pensée lui-même, où elle est indiquée comme l'un des principes organisationnels du système conceptuel humain. En ce sens, la métaphore est définie comme la compréhension d'un concept ou d'un domaine conceptuel à l'aide d'un autre concept, c'est-à-dire, d'un domaine conceptuel. Le mécanisme de la métaphore consiste à cartographier la structure d'un concept, généralement sensoriel et bien connu, vers un autre domaine. Dans la linguistique cognitive, le sens est assimilé à la conceptualisation - la conceptualisation implique la formation et la compréhension de concepts basés sur « l'expérience physique, sensorielle, 
émotionnelle et intellectuelle d'une personne avec le monde qui l'entoure » (Lakoff, Johnson, 1980 : 178-198 ; Klikovac, 2004 : 9).

Dans notre corpus, le sexe féminin se conceptualise comme :

A) UN BATIMENT : une étable, une boutique, банка, кућa où on entre et sort pour réaliser de différentes activités. Ce va-et-vient fait allusion au coït. La conceptualisation au niveau inférieure provient de la métaphore supérieure AMOUR EST EDIFICE. Il est à noter que ces termes renvoient aussi au vagin grand et large. Quant au mot boutique, la métaphore se base aussi sur le concept AMOUR EST COMMERCE, ce que montre l'exemple suivant : "Bien souvent à telle pratique les femmes ouvrent leur boutique »; " Oh ! ma vie, venez ici, et fermez la boutique, c'est aujourd'hui la fête » (Guiraud, 1995 : 187).

B) UN LIEU SACRE : un temple, un sanctuaire, une chapelle, un autel où l'on trouve un plaisir spirituel, physique et une grâce corporelle quand on y pénètre. La métaphore du niveau supérieur repose sur AMOUR EST RELIGION.

C) UN LIEU FORTIFIÉ : une forteresse, une citadelle, une casemate. Dans notre cas, la métaphore supérieure renvoie au concept AMOUR EST COMBAT au sein duquel le vagin s'aperçoit comme un endroit d'importance et de valeur pour les conquérants qu'ils désirent occuper à tout prix.

D) UNE PIÈCE : une chambre, un cabinet, une cuisine, un atelier. Ces exemples reflètent que le vagin est appréhendé comme une place intégrée dans le cadre d'un édifice où les gens séjournent et font leur travail, d'où provient la métaphore supérieure AMOUR EST ACTIVITÉ.

E) UN ANIMAL : un chat, une chatte, un angora, un faucon, чавка. Une telle conceptualisation réside dans le fait que le vagin est « vorace » et « dévore » le pénis comme ces bêtes qui avalent leur proie.

F) UNE PLANTE : un abricot, une figue $e^{18}$, un nénuphar, смоква. Le trait commun de ces plantes renvoyant au sexe de la femme est la jutosité et l'humidité.

G) UN ESPACE GÉOGRAPHIQUE : Pays-Bas. Le sexe féminin peut être conceptualisé comme un pays ou un état. Dans notre exemple, c'est l'adjectif

${ }^{18}$ Le caractère obscène de ce mot s'explique par le fait qu'il entre dans le cadre de la locution «faire la figue à qqn » (se moquer) qui est un calque de l'italien « far la fica », geste vulgaire de dérision, la fica représentant le sexe de la femme, sens repris du grec et passé en français (Rey, $2006: 1425)$. 
bas qui implique le contenu obscène de ce syntagme nominal étant donné que le vagin est situé en partie basse du ventre.

Ainsi, beaucoup d'exemples de notre corpus indiquent que le sexe de la femme est conceptualisé comme un contenant de liquide, soit de l'eau froide (fosse, bouteille, puits, vase), soit d'une substance chaude (pot, chaudron, marmite).

\section{CONCLUSION}

Le vocabulaire de la sexualité se différencie par une richesse verbale remarquable et lui attribue un statut particulier dans le système de la langue. Le moindre concept est représenté par des nombreux mots alternatifs, c'est-à-dire des synonymes. Dans la langue française nous avons identifié 108 mots représentant le sexe de la femme tandis qu'en serbe, on a 33 exemples. Il s'agit des mots qui sont non-obscènes au niveau du signifiant mais qui, au niveau du signifié sont dotés d'un contenu obscène. Dans les deux langues, du point de vue de la métaphore lexicale, le plus grand nombre d'exemples montrent que le vagin s'identifie aux objets ronds et ovales à travers l'analogie de forme: il est un vaisseau, un ustensile creux, un creux naturel, une ouverture. Moins nombreuses sont les occurrences relevant d'une analogie de fonction et de couleur. À ce titre, le sexe de la femme est perçu comme une pièce à l'intérieur de laquelle on pénètre et dont la nature varie d'après le contexte et la nature de l'occupant ou comme un instrument émettant la lumière. Quant aux sèmes de l'expression collective, on conclut qu'en français et en serbe les zoonymes, les phytonymes et les noms propres font référence aux humains et à leurs parties du corps, dans notre cas, au vagin. En ce qui concerne la métaphore conceptuelle, le sexe féminin se conceptualise aussi comme un bâtiment, un lieu, une place fortifiée, une pièce, un animal, une plante, un espace géographique ou comme un contenant de liquide rempli de la matière froide ou chaude. Dans les deux langues, dans un certain nombre d'occurrences, on a dégagé les mêmes synonymes, ce qui montre que le concept du vagin est translinguistique, transculturel et transnational. Autant de noms désignant le vagin nous dévoile que dans les deux langues et cultures son culte est soigneusement nourri et que toutes les activités de l'homme, son entourage naturel, ses actions, ses modes de vie, son comportement, son humeur, son plaisir spirituel et charnel s'articulent sans équivoque autour du sexe féminin et de l'acte sexuel et représentent une source inépuisable de l'inspiration de vivre. Ainsi, ils continueront à façonner son avenir. La différence s'affiche en termes de 
l'origine des mots alternatifs : en français, les synonymes du vagin proviennent de la littérature latine et de la littérature médiévale française tandis qu'en serbe, les synonymes relatifs au sexe de la femme sont issus plutôt du langage argotique serbe.

Ivan Jovanović

\section{ALTERNATIVE NAMES OF THE FEMALE SEXUAL ORGAN IN FRENCH AND SERBIAN: LEXICO-SEMANTIC ASPECT}

\section{Summary}

This article is an attempt to provide an analysis of alternative names of the female sexual organ in French and Serbian with the aim to show all the similarities and differences that occur at the lexico-semantic level. Starting from the postulates of semantic and conceptual analyses as well as from Guiraud's (1995) classification of obscene lexical units, our corpus comprises forms of polysemy that induce the meaning of alternative names (metaphorical relations based on form, function, color and schemes of collective expression; metonymic relations based on part vs. all) and the types of conceptual metaphors based on which the female sexual organ is conceptualized as a building, a place, an animal, a plant or a geographic space.

The corpus of our research has been compiled based on the French and Serbian dictionaries of obscene words by Pierre Guiraud (1995), Alfred Delvau (1997), Robert Édouard (2004), Danko Šipka (2011), Petrit Imami (2007), as well as the dictionaries of slang by Abdelkarim Tengour (2013), Dragoslav Andrić (1976) and Borivoje Gerzić (2010).

Key words: alternative names, feminine gender, semantics, French language, Serbian language.

\section{RÉFÉRENCES BIBLIOGRAPHIQUES}

Andrić, D. (1976). Rečnik žargona. Beograd : BIGZ (Андрић, Д. (1976). Речник жаргона. Београд : БИГЗ.)

Delvau, A. (1997). Dictionnaire érotique moderne. Paris : Domaine français.

Édouard, R. 1983. Dictionnaire des injures. Paris : Sand et Tchou.

Gortan-Premk, D. (2004). Polisemija i organizacija leksičkog sistema u srpskom jeziku. Beograd : Zavod za udžbenike i nastavna sredstva. (Гортан-Премк, Д. (2004). Полисемија и организација лексичког система у српском језику. Београд : Завод за уџбенике и наставна средства.)

Gerzić, B. (2012). Rečnik srpskog žargona. Beograd : SA.

Guiraud, P. (1975). Les gros mots. Paris : Presse universitaire de France. 
Guiraud, P. (1978). La sémiologie de la sexualité. Paris : Payot. Guiraud, P. (1995). Dictionnaire érotique. Paris : Grand Bibliothèque Payot. Imami, P. (2007). Beogradski frajerski rečnik. Beograd : NNK International.

Jovanović, I. (2019). Upotreba i značenje opscene leksike u srpskim novokomponovanim pesmama. In : Marković, J. (ed.) (2019). Opscena $i$ druga kolokvijalna leksika u srpskom i makedonskom jeziku. Niš : Filozofski fakultet. 153 - 176. (Јовановић, И. (2019). Употреба и значење опсцене лексике у српским новокомпонованим песмама. In : Марковић, J. (ed.) (2019). Опсцена и друга колоквијалналексика у српском и македонском језику. Ниш : Филозофски факултет. 153 - 176.)

Klikovac, D. (2004). Metafore u mišljenju i jeziku. Beograd : Biblioteka XX vek. Kovačević, M. (1999). Stilistika i gramatika stilskih figura. Kragujevac : Kantakuzin. (Ковачевић, М. (1999). Стилистика и граматика стилских фигура. Крагујевац : Кантакузин.)

Lagail, A.S. (1903). Les Paradis charnels ou Le divin bréviaire des amants. Paris: Priape ville.

Lakoff, G.-Johnson, M. (1980). Metaphors We live by. Chicago : University of Chicago Press.

Le Petit Larousse (2010). Paris : Larousse.

Popović, M. (2009). Leksička struktura francuskog jezika: morfologija i semantika. Beograd : Zavod za udžbenike i nastavna sredstva. (Поповић, М. (2009). Лексичка структура француског језика: морфологија и семантика. Београд : Завод за уџбенике и наставна средства.)

Rey, A. (2006). Dictionnaire historique de la langue française, t. 1-3, Paris : Le Robert.

Stanković, S. (2012). Iz kontrastivne analize jezika: problemi, rezultati, klasifikacija i primena. Philologia Mediana, 4, 381-391. (Станковић, С. (2012). Из контрастивне анализе језика: проблеми, резултати, класификација и примена. Philologia Mediana, 4, 381-391.)

Tamba-Metz, I. (2012). La Sémantique. Paris : PUF.

Tengour, A. (2013). Tout l'argot des banlieues. Le dictionnaire de la zone. Paris : Opportun.

Touratier, Ch. (2010). La Sémantique. Paris : Armand Colin.

Ullmann, S. (1952). Précis de sémantique française. Berne : Francke.

Šipka, D. (2011). Rečnik opscenih reči i izraza. Novi Sad : Prometej. (Шипка, Д. (2011). Речник опсцених речи и израза. Нови Сад : Прометеј.) 
